\title{
Practice and preferences of endotracheal intubation by emergency and critical care physicians in Riyadh, Kingdom of Saudi Arabia
}

\author{
Butt TS $^{1 *}$, AlShuduki $A^{2}$, Kalla M ${ }^{3}$, Amani $^{2}$ and MN Qureshi ${ }^{4}$ \\ ${ }^{1}$ Chairman, Emergency Medicine, KFSHRC-Madinah, Kingdom of Saudi Arabia \\ ${ }^{2}$ Resident, Emergency Medicine, KFSH\&RC-Riyadh, Kingdom of Saudi Arabia \\ ${ }^{3}$ Consultant, Emergency Medicine, KFSH\&RC-Madinah, Kingdom of Saudi Arabia \\ ${ }^{4}$ Consultant, Emergency Medicine, KFSH\&RC-Riyadh, Kingdom of Saudi Arabia
}

\begin{abstract}
Purpose: This survey aimed to identify the practice and preferences of endotracheal intubation amongst Emergency and Critical Care physicians in Riyadh, Kingdom of Saudi Arabia (KSA).

Methods: An electronic questionnaire was created and distributed using SurveyMonkey ${ }^{\circledR}$. This was sent to emergency and critical care physicians working at various hospitals in Riyadh, KSA. Data was collected regarding their airway skills, experience in performing the procedure, drugs of choice for rapid sequence intubation, the availability of difficult airway equipment and participant demographics.

Results: Out of the 210 physicians contacted we received 100 completed responses. Of these two thirds were Emergency Medicine physicians or residents. Preoxygenation is performed $74 \%$ of the time and direct laryngoscopy is preferred over video laryngoscopy. Doctors with less than 5 years of clinical experience are more likely to perform airway assessments prior to the procedure compared to the more experienced clinicians; $32 \%$ of the respondents are always performing airways assessment. $84 \%$ of respondents are working in clinical areas which have a difficult airway cart available. Etomidate and succinylcholine are the most commonly used induction and paralytic agents.

Conclusion: While pre-oxygenation is performed prior to the majority of the intubations, airway assessment is not routinely performed. Junior doctors are more inclined to perform airway assessment. Direct laryngoscopy is the preferred method of endotracheal intubation by the majority. The use of induction and paralytic agents vary widely. Difficult airway carts are not universally available.
\end{abstract}

\section{Introduction}

Critically ill patients that present to the Emergency Department (ED) or Intensive Care Unit (ICU) are physiologically unstable and may have poor reserves to sustain life. These patients may require Endotracheal Intubation (ETI) to secure their airways and provide ventilator support [1-3]. Outside the operating rooms, ETI is most commonly performed by the Emergency Physicians (EPs) in the ED and the Critical Care Physicians (CCPs) in the ICU [4].

Clinicians at the forefront of managing these acutelydecompensating patients need to be proficient with airway management skills, have a complete knowledge of the equipment, and have formal training to perform the procedure [5]. The intubating physician has to have access to airway equipment, be able to recognize and intervene in a timely and effective way to manage any complications [6]. The intubating physician should also be knowledgeable in the use of the rescue devices which should be available universally [7].

ETI protocols are established by various airway management societies to guide the clinical practice. This survey was conducted to understand, the current practice of ETI among the emergency and critical care physicians working at various hospitals in Riyadh, Kingdom of Saudi Arabia (KSA).

\section{Methods}

An electronic survey questionnaire was designed to study the practice of intubation preferences and techniques of Emergency and Critical Care Physicians. The questionnaire was distributed to the physicians using SurveyMonkey ${ }^{\circ}$. Data collected were participant demographics, intubation procedure preference, induction and paralytic agent of choice, and the availability of rescue and emergency intubation equipment. The questionnaire was distributed amongst attending and resident physicians, working in ED and ICU setting in various hospitals in Riyadh, KSA.

The data collected was analyzed using IBM- SPSS V27 program. Descriptive statistics, cross-tabs, and Chi-square were performed to correlate between categorical variables to check for associations.

\section{Results}

The questionnaire was sent to 210 Physicians. A hundred questionnaires (48\%) were completed and returned. Out of the total

${ }^{*}$ Correspondence to: Taimur S. Butt, MD, Chairman, Emergency Medicine, KFSHRC-Madinah, Kingdom of Saudi Arabia, E-mail: tbutt@kfshrc.edu.sa

Received: March 12, 2021; Accepted: April 12, 2021; Published: April 16, 2021 
respondents, $91 \%$ were residents, and $9 \%$ were attending physicians. EPs and emergency medicine residents constituted $67 \%(67 / 100)$ of the total respondents. Two respondents had no prior experience in ETI, and one respondent was unsure of how to intubate. $81 \%$ of respondents have less than five years'in clinical practice (Table 1).

Airway assessment is performed always by $32 \%$ of the responders and sometimes by $35 \%$ (Figure 1). Pre-oxygenation is always performed by $74 \%$ of the respondents, while $26 \%$ reported pre-oxygenating most of the time or sometimes (Figure 2). Direct laryngoscopy is the preferred method of intubation for $74 \%$ of the respondents, while only $26 \%$ preferred video-laryngoscopy. All the respondents used endotracheal tube introducers or stylets as adjuncts. The stylet was the adjunct of choice for $86 \%$ of the respondents, $23 \%$ used a stylet or bougie, $13 \%$ used a bougie only.

Etomidate is the most commonly used (62\%) induction drug choice by the responders. Of these, $24 \%$ used Etomidate exclusively. Propofol is used by $54 \%$, followed by Ketamine $46 \%$ and Midazolam $27 \%$ of the total responders (Figure 3). Suxamethonium was the preferred paralyzing agent for $47 \%$ of respondents, while $53 \%$ preferred rocuronium on its own or in combination. Of these 37 percent used rocuronium exclusively; $15 \%$ used either suxamethonium or vecuronium.

Out of the total 100 responses, $89 \%$ of respondents have access to video laryngoscope. However, only $26 \%$ use this and the rest $74 \%$ use direct laryngoscope. Out of the remaining respondents, $5 \%$ were unsure of the availability of video laryngoscope, and $6 \%$ do not have video laryngoscope available at all. $84 \%$ of the respondents have difficult airway carts available. Four percent of respondents did not have a difficult airway cart while $12 \%$ have never checked for the availability of a difficult airway cart (Table 2).

\section{Discussion}

Our data show significant variance in the practice of ETI among EM and CCP in the city of Riyadh and consequently a potential impact on standardization of the clinical practice. Airway management training is mandatory for all EM and CCPs during residency. In most countries, including the KSA, trainees in the above specialties are expected to

Table 1. Years of experience vs number of respondents

\begin{tabular}{|l|c|}
\hline $\begin{array}{l}\text { Level of training and/ or } \\
\text { Years of Experience }\end{array}$ & Number of respondents N (\%) \\
\hline R1 & $21(21 \%)$ \\
\hline R2 & $23(23 \%)$ \\
\hline R3 & $23(23 \%)$ \\
\hline R4 & $14(14 \%)$ \\
\hline 5 years' experience & $10(10 \%)$ \\
\hline 7 years' experience & $1(1 \%)$ \\
\hline 10 or more years' experience & $8(8 \%)$ \\
\hline
\end{tabular}

Table 2. Availability of video laryngoscope and difficult airway cart

\begin{tabular}{|l|c|c|c|c|c|}
\hline & \multicolumn{5}{|c|}{ Availability of Video Laryngoscope } \\
\hline \multirow{2}{*}{$\begin{array}{l}\text { Availability of } \\
\text { Difficult Airway } \\
\text { Cart in your } \\
\text { Department }\end{array}$} & $\begin{array}{c}\text { Never } \\
\text { Checked }\end{array}$ & No & Yes & Total \\
\cline { 2 - 6 } & Checked & 1 & 2 & 9 & $\mathbf{1 2}$ \\
\cline { 2 - 6 } & No & 0 & 1 & 3 & $\mathbf{4}$ \\
\hline Yes & 4 & 3 & 77 & $\mathbf{8 4}$ \\
\hline Total & & 5 & 6 & 89 & 100 \\
\hline
\end{tabular}

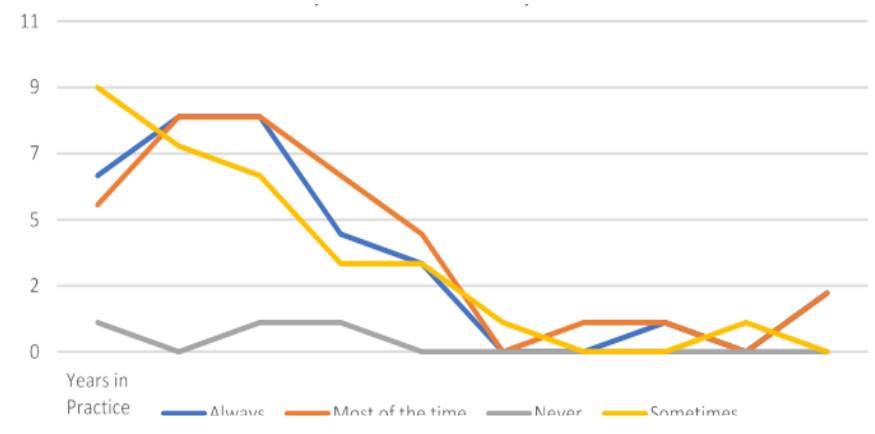

Figure 1. Years of Experience vs Airway assessment

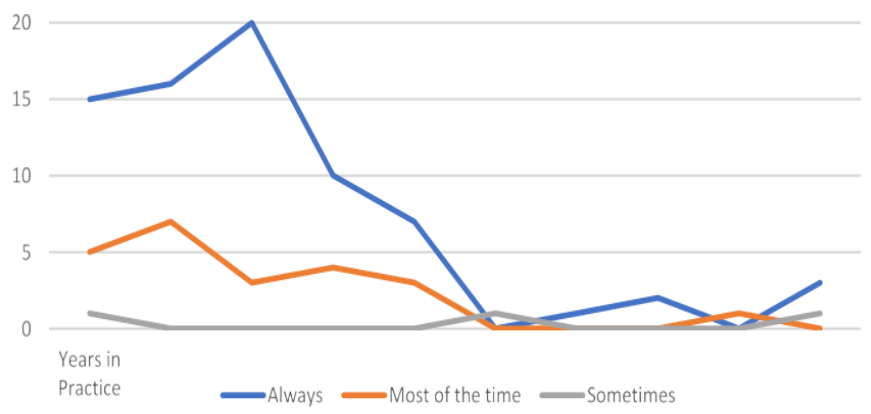

Figure 2. Years in practice vs performing pre-oxygenation

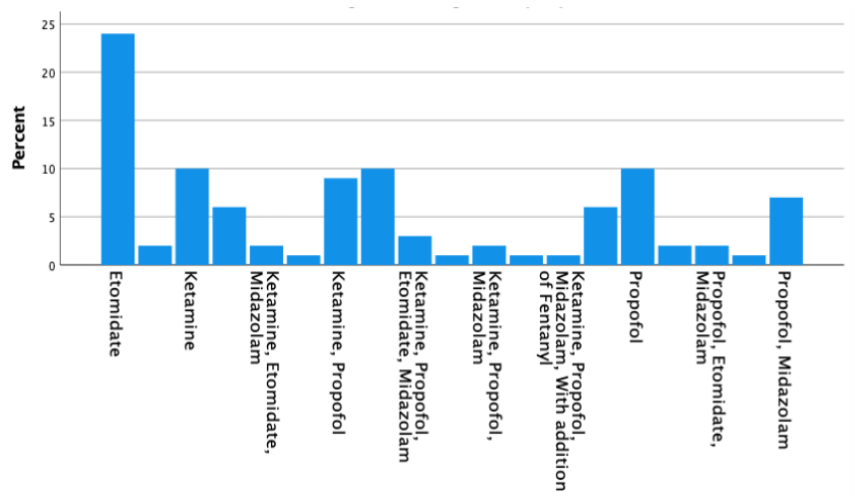

Figure 3. Use of induction agent

learn and perform unsupervised ETI to complete their structured training programs.

ETI is a high-risk, lifesaving advanced airway skill, performed in the hospitals and prehospital setting by physicians and or allied health staff $[2,4,8]$. Admitted patients in need of advanced airway and ventilation, are usually managed by the CCPs. Correspondingly, the teaching of this skill and receptiveness of a trainee becomes challenging when there is a lack of adherence to the standard protocols $[7,9,10]$. Teaching and skills development also depends on the locally available resources. However, the practice of intubation varies widely across different countries [11]. Maintaining post training competency is also a challenge $[9,10]$.

Pre-oxygenation and airway assessment are critical steps before intubation to mitigate potential difficulties and complications [8]. Adequate pre-oxygenation during a Rapid Sequence Intubation (RSI) can be achieved within 3-5 minutes through a non rebreather mask. This would increase the safe apnea time by nitrogen washout, improved 
oxygen delivery and increased uptake of alveolar oxygen into the blood. It is a standard of care that may increase desaturation time up to 8 minutes [8,12]. Our survey showed that not all intubators pre-oxygenate their patients; only $74 \%$ always pre-oxygenated. Airway assessment is also not performed by all participants. Generally, junior doctors have a higher propensity to perform airway evaluation. However, the more senior the respondent was the more likely they did not perform an airway assessment. This may show a lack of standardization or operator overconfidence.

The choice of induction and paralyzing agents during RSI may vary widely $[13,14]$. This can be due to a local protocol, situational needs, experience, and confidence in the use of a particular drug, etc. Midazolam may induce hypotension during ETI. However, $27 \%$ of our surveyed physicians use it in their practice. Recently, Ketamine is becoming a more favorable induction agent, recommended due to its stable hemodynamic properties $[15,16]$. However, Etomidate and Propofol are other commonly used induction agents.

Video Laryngoscopy (VL) is being advocated as the standard for difficult airway intubation but its superiority for first-pass intubation is not conclusive [17-20]. ETI risk awareness, availability of equipment and teaching during training programs continues. However, standardization of difficult airway carts is still lacking [21]. Our survey findings were similar to Cook and Kelly that difficult airway or rescue equipment is not available all the time [22,23].

Health Care Workers (HCW) at the frontline are at the highest risk of occupational exposure to COVID-19 infection. Since, the advent of the COVID-19 outbreak, VL is being supported as a safer procedure compared to DL $[21,20]$. This has resulted globally in HCW changing their approach to "self-safety" and he alth promotion. Among our respondents despite having the facility, majority preferred direct laryngoscopy. It is not clear whether this is a lack of experience, equipment knowhow or a personal choice. The use of VL is now promoted as the method of choice to perform ETI [24,25].

At our institution, we have standardize the difficult airway adjuncts utilized during ETI. A difficult airway algorithm is proposed for patients with difficult or anticipated difficult airways (Figure 4). This poster is displayed in the resuscitation room as a visual clue for the intubating team. The bidirectional bottom arrow is to allow flexibility to the operator. As at times, switching between VL and DL may improve success rate. Each resuscitation room in our institution is equipped with a difficult airway cart. This cart is always stocked with different airway adjuncts including various types and sizes of DL and VL blades, bougies, stylets, supraglottic airway devices including LMA ${ }^{\oplus}$ (laryngeal mask airway) I-gel ${ }^{\oplus}$, fiber-optic or video bronchoscope, No.10 blade

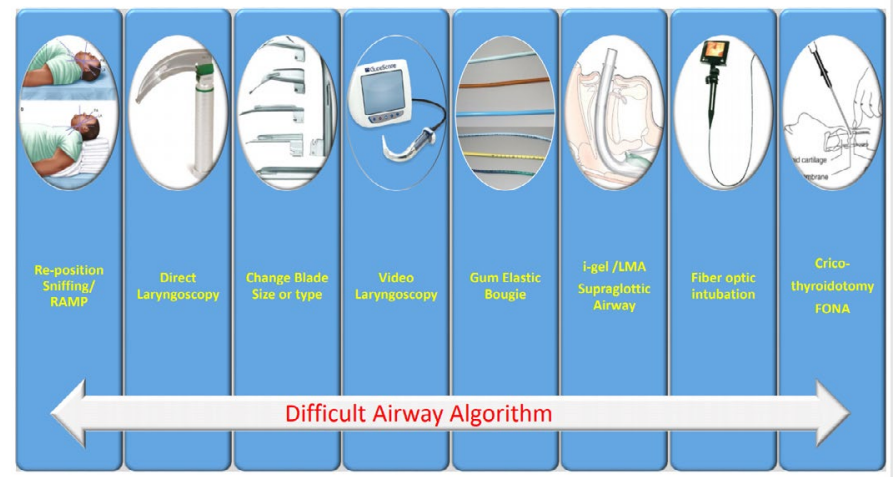

Figure 4. Difficult airway algorithm and a formal front of neck access (FONA) kit. This practice facilitates the best possible outcome.

\section{Conclusion}

Our survey shows that there is a significant disparity among the physicians in managing ETI procedure. DL remains the preferred method of ETI by the majority. While pre-oxygenation is performed prior to the majority but not before all the ETIs. Junior doctors are more likely to perform airway assessment prior to intubation. Etomidate and succinylcholine are the most commonly used induction and paralytic agents. However, there is a need for standardization, universal availability of VL, and difficult airway carts.

\section{Recommendations}

The authors recommend that practitioners at all levels should follow a standardized approach to intubating a patient. There should be a rigorously structured training program for trainees to develop competencies in all available airway adjuncts including VL. To improve patient safety, all patients must be adequately pre-oxygenated before the RSI attempts. To improve HCW safety, healthcare facility must provide staff access to VL and implement protocols for intubating patients with COVID-19 infections.

\section{Limitations}

Our study was limited to physicians in Riyadh, thus the results may not be generalizable. The majority of the respondents had 5 years or less experience, this has potentially skewed the results. The survey did not explore the clinical scenarios that may dictate the choices made. There is a need for a larger prospective study.

\section{Disclosure}

None.

\section{References}

1. A.J, Oglesby. "Should etomidate be the induction agent of choice for rapid sequence intubation in the emergency department?" Emergency Medicine Journal 21 (2004): 655-659.

2. B.Y, Gravesteijn, et al. "Variation in the practice of tracheal intubation in Europe after traumatic brain injury: a prospective cohort study." Anaesthesia 2020, 75 (2020): 45-53.

3. Baek, Moon Seong, Myong Ja Han and Jin Won et al. Huh. "Video laryngoscopy versus direct laryngoscopy for first-attempt tracheal intubation in the general ward." Annals of Intensive Care 8.1 (2018).

4. Brewster, D. J., et al. "Consensus statement: Safe Airway Society principles of airway management and tracheal intubation specific to the COVID-19 adult patient group." The Medical Journal of Australia (2020): Preprint only.

5. Brown, C.A, et al. "Pragmatic recommendations for intubating critically ill patients with suspected COVID-19." Journal of the American College of Emergency Physicians Open (2020): 80-84.

6. C, Morris, et al. "Anaesthesia in haemodynamically compromised emergency patients: does ketamine represent the best choice of induction agent?" Anaesthesia 64 (2009) 532-539.

7. C, Reid, et al. "The who, where, and what of rapid sequence intubation: Prospective observational study of emergency RSI outside the operating theatre." Emergency Medicine Journal 21.3 (2001): 296-301

8. C.A, Graham. "Advanced airway management in the emergency department: what are the training and skills maintenance needs for UK emergency physicians?" Emergency Medicine Journal 21 (2004): 14-19.

9. Collins, S.R. "Direct and indirect laryngoscopy: Equipment and techniques." Respiratory Care 59.6 (2014): 820-864.

10. Cook, T. M., Macdougall-Davis, S. R. "Complications and failure of airway management." British Journal of Anaesthesia 109.SUPPL1 (2012): 68-85. 
11. De Jong, A, B Jung and S Jaber. "Intubation in the ICU: we could improve our practice." Critical Care 18.209 (2014).

12. Dost, Burhan, Ersin Koksal and Özlem et al. Terzi. "Attitudes of Anesthesiology Specialists and Residents toward Patients Infected with the Novel Coronavirus (COVID-19): A National Survey Study." Surgical Infections 21.4 (2020): 349-355.

13. G.D, Simpson, et al. "Tracheal intubation in the critically ill: a multi-centre national study of practice and complications." British Journal of Anaesthesia 108.5 (20112): 792-799.

14. G.1, Bouroche and Bourgain J.L. "Preoxygenation and general anesthesia: a review." Minerva Anestesiologica 81.8 (2015): 910-920.

15. J.A, Law, et al. "The difficult airway with recommendations for management - Part 1 Difficult tracheal intubation encountered in an unconscious/induced patient." Canadian Journal of Anesthesia 60.11 (2013): 1089-1118.

16. J.M, Mosier, et al. "Tracheal Intubation in the Critically Ill. Where We Came from and Where We Should Go." American Journal of Respiratory and Critical Care Medicine 201.7 (2020).

17. L, Pillay and Hardcastle T. "Collective Review of the Status of Rapid Sequence Intubation Drugs of Choice in Trauma in Low- and Middle-Income Settings (Prehospital, Emergency Department and Operating Room Setting)." World Journal of Surgery 41 (2017): 1184-1192.
18. M, Martin, et al. "Nationwide survey on training and device utilization during tracheal intubation in French intensive care units." Annals of Intensive Care 10.2 (2020).

19. Meng, L, et al. "Intubation and Ventilation amid the COVID-19 Outbreak: Wuhan's Experience." Anesthesiology 132.6 (2020): 1317-1332.

20. Nimmagadda, U, et al. "Preoxygenation: Physiologic basis, benefits, and potentia risks." Anesthesia and Analgesia 124.2 (2017): 507-817.

21. Sollid, S. J.M., J. Mellin-Olsen and T. et al. Wisborg. "Emergency airway management - by whom and how?" Acta Anaesthesiologica Scandinavica 60.9 (2016): 1185-1187.

22. T, Edwards, et al. "Prehospital use of ketamine and midazolam in an urban advanced paramedic practitioner service: a retrospective review." Emergency Medicine Journal 33.e8 (2016)

23. T.M, Cook, Woodall $\mathrm{N}$ and Benger H.J. "Major complications of airway management in the UK: results of the Fourth National Audit Project of the Royal College of Anaesthetists and the Difficult Airway Society. Part 2: intensive care and emergency departments $\uparrow . "$ British Journal of Anaesthesia 106.5 (2011): 632-642.

24. T.M, TCook and Kelly F.E. "A national survey of videolaryngoscopy in the United Kingdom." British Journal ofAnaesthesia 118.4 (2017): 593-600.

25. Yao, W, et al. "Emergency tracheal intubation in 202 patients with COVID-19 in Wuhan, China: lessons learnt and international expert recommendations." British Journal of Anaesthesia 125.1 (2020): e28-e37.

Copyright: (C2021 Butt TS. This is an open-access article distributed under the terms of the Creative Commons Attribution License, which permits unrestricted use, distribution, and reproduction in any medium, provided the original author and source are credited. 